\title{
Recurrent retroperitoneal inflammatory myofibroblastic tumor: A case report
}

\author{
XINJUN WANG ${ }^{1}$, XIAOKUN ZHAO $^{2}$, JOSEPH CHIN $^{3}$, LIANG ZHU $^{2}$, ZIJIAN WANG $^{2}$ and ZHAOHUI ZHONG ${ }^{2}$ \\ ${ }^{1}$ Department of Urology, Zhongshan Hospital, Xiamen University, Xiamen, Fujian 361004; ${ }^{2}$ Department of Urology, \\ The Second Xiangya Hospital, Central South University, Changsha, Hunan 410011, P.R. China; \\ ${ }^{3}$ Department of Surgery, Division of Urology, London Health Sciences Centre, \\ University of Western Ontario, London, ON N6A 3K7, Canada
}

Received August 26, 2014; Accepted June 7, 2016

DOI: $10.3892 / 01.2016 .4767$

\begin{abstract}
Inflammatory myofibroblastic tumor (IMT), which was previously known as inflammatory pseudotumor, is characterized by myofibroblastic spindle cells accompanied by inflammatory infiltrates. IMT is a rare lesion of unclear etiology, which induces non-specific clinical symptoms. The present case report describes a 74-year-old female patient with recurrent IMT, which was successfully re-resected 30 months subsequent to initial surgical removal. The patient presented with left hydroureteronephrosis and a $10-\mathrm{cm}$ paravertebral mass which, upon surgery, was found to involve the descending colon. Successful, complete en bloc re-resection was achieved, and at 24 months follow-up, the patient remained clinically free of disease. Complete surgical extirpation, where feasible, is the recommended treatment for primary and recurrent IMT lesions.
\end{abstract}

\section{Introduction}

Inflammatory myofibroblastic tumor (IMT) was formerly known as inflammatory pseudotumor, and is a lesion characterized by myofibroblastic spindle cells accompanied by inflammatory infiltrates. Although the lung is the most common site of occurrence, IMT may arise in diverse extra-pulmonary locations. The etiology of IMT is unclear; however, the anaplastic lymphoma receptor tyrosine kinase gene may play a role in its pathogenesis (1). The clinical presentation of IMT is non-specific and may include fever, weight loss, malaise and anemia. Retroperitoneal IMT usually grows slowly and may present as a solid abdominal mass, accompanied by abdominal pain and weight loss. Gastrointestinal and urinary symptoms

Correspondence to: Dr Zhaohui Zhong, Department of Urology, The Second Xiangya Hospital, Central South University, 139 Ren Min Road, Changsha, Hunan 410011, P.R. China

E-mail: jzhonguro@126.com

Key words: retroperitoneal inflammatory myofibroblastic tumor, recurrent, surgery may occur with an enlarging expansile mass (2). There are no specific radiographical features for IMT. For abdominal IMT, a solid mass abutting and compressing various organs is the usual presentation. Retroperitoneal IMT is rare, with only 12 cases reported in English literature to date (2-10). The present study describes a case of retroperitoneal IMT, which recurred 30 months following initial surgery, and where re-resection was successfully performed. Written informed consent was obtained from the patient for the publication of the present study.

\section{Case report}

A 74-year-old female initially presented with a $10-\mathrm{cm}$ retroperitoneal mass, discovered incidentally during cholecystectomy. The mass was resected via a midline transperitoneal incision. Histopathological examination identified the lesion as IMT. Patient history included a left pulmonary lobectomy for bronchiectasis 20-years previously, and hypertension for 40-years. Subsequently, the patient presented with a palpable left lower-quadrant abdominal mass 30 months later. Hematological and biochemical parameters, including carcinoembryonic antigen and $\alpha$-fetoprotein expression, were all within normal limits. Abdominal computerized tomography (CT) with contrast revealed an $8 \times 7.5 \times 8.5 \mathrm{~cm}$, well-defined heterogeneous mass with uneven enhancement (Fig. 1), associated with left hydroureteronephrosis. There was no regional lymphadenopathy. Metastatic workup, including a chest CT scan and radionuclide bone scan, was negative.

Following multidisciplinary consultation, laparotomy was performed via a left subcostal incision. A $10-\mathrm{cm}$ mass was identified, which was compressing the kidney and proximal ureter and adherent to the descending colon. The kidney and ureter were successfully dissected free but partial left colectomy was required to facilitate en bloc removal of the tumor mass. The postoperative course was uneventful. Gross histopathological examination revealed the tumor to be encapsulated, with a grey-whitish cut surface (Fig. 2). Microscopically, the tumor demonstrated proliferation of spindle cells and infiltration of lymphocytes, plasma cells and eosinophils (Fig. 3). Immunohistochemistry revealed diffuse positivity for vimentin, CD68 and desmin; focal staining for 


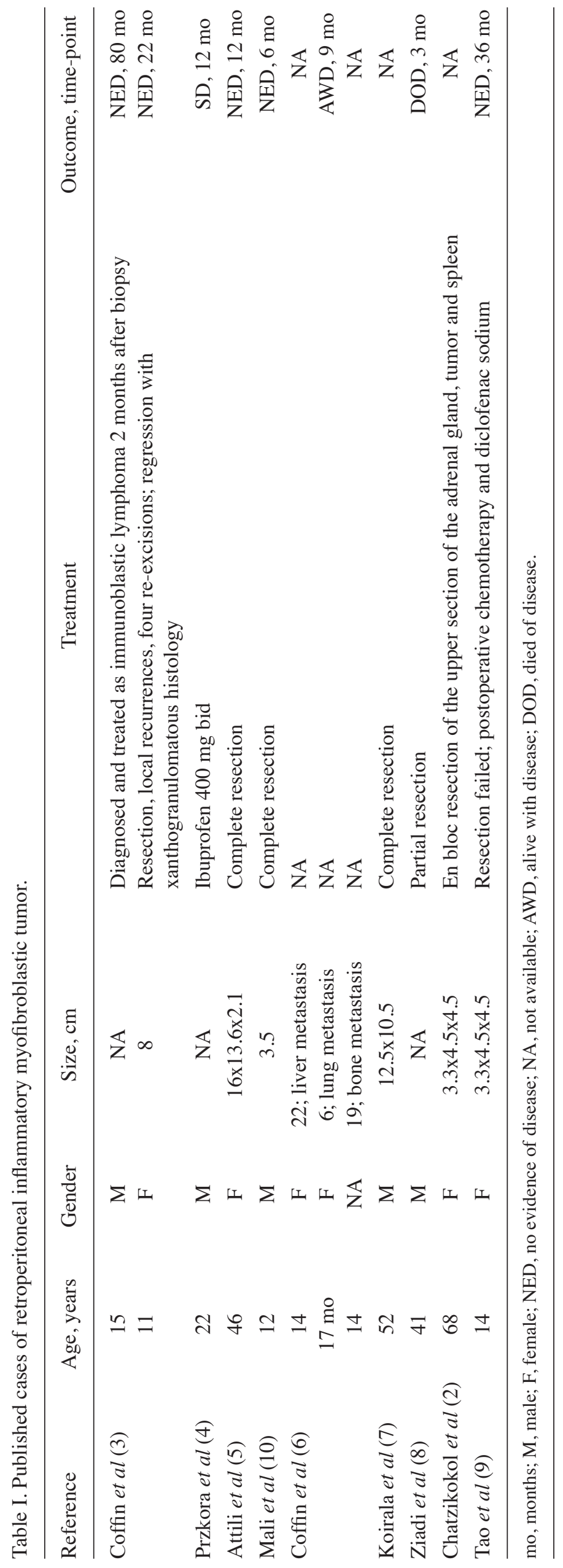




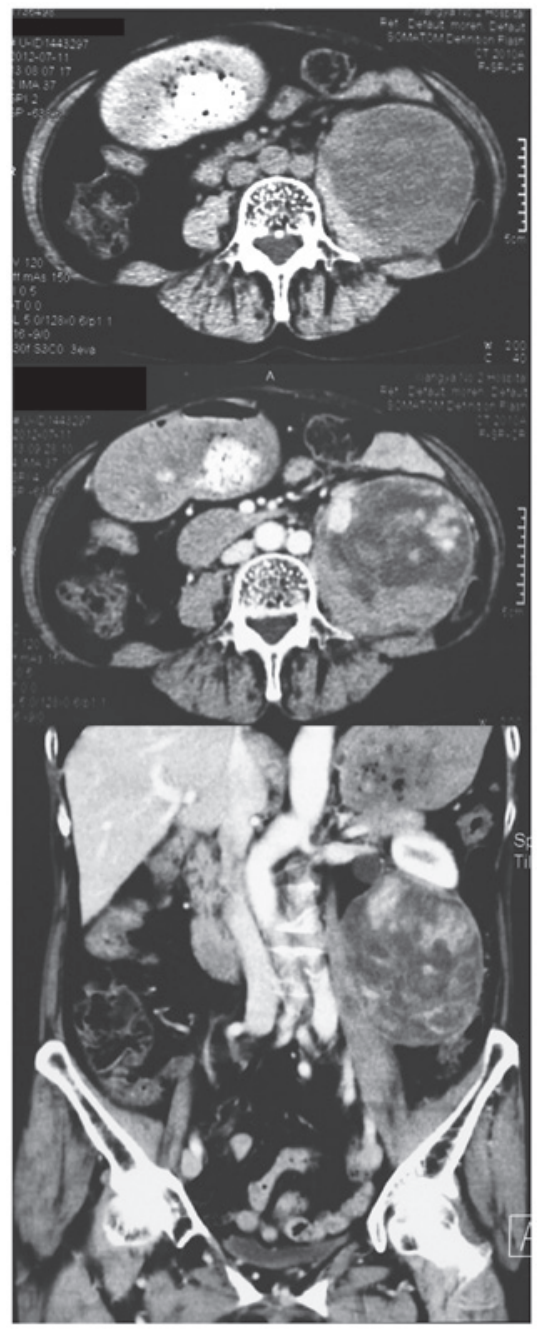

Figure 1. Computed tomography scan revealed a well-defined, heterogeneous lesion with uneven enhancement, occupying the left retroperitoneal region and measuring $\sim 7.5 \times 8.5 \times 8 \mathrm{~cm}$.

myogenin and Ki-67; and negativity for CD34, cytokeratin, smooth muscle actin and S100.

The multidisciplinary treatment team did not recommend postoperative adjuvant therapy. The patient has remained free of local or distant recurrence 24 months subsequent to re-resection, at the time of the present report.

\section{Discussion}

Inflammatory myofibroblastic tumor (IMT) is a distinct lesion, composed of myofibroblastic spindle cells with intermediate biological potential, accompanied by an inflammatory infiltration of plasma cells, lymphocytes and eosinophils $(5,6)$. The most common sites of IMT are the lungs and soft-tissue viscera in children and young adults. The etiology is unclear, although trauma, surgery, inflammation and infection by Epstein-Barr virus or human herpes virus have been proposed as potential causative factors (11). Furthermore, chromosomal rearrangement involving the ALK gene results in activation of a tyrosine kinase receptor and may induce abnormal expression. Immunohistochemically, $50 \%$ of IMTs are positive for ALK (12), suggesting that the ALK gene may have a role in the pathogenesis of IMT (1).

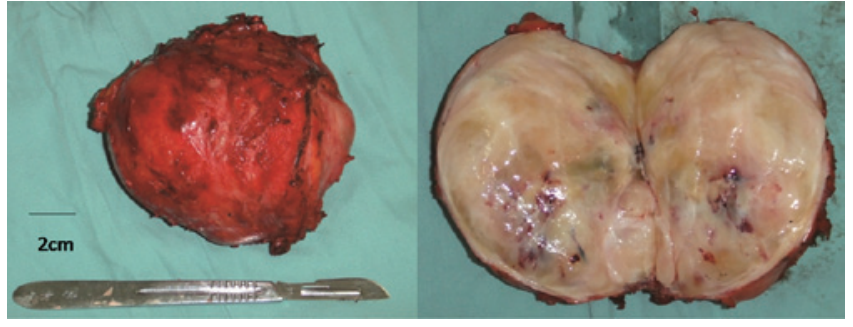

Figure 2. The resected tumor was an encapsulated mass with a grey-whitish cut surface.

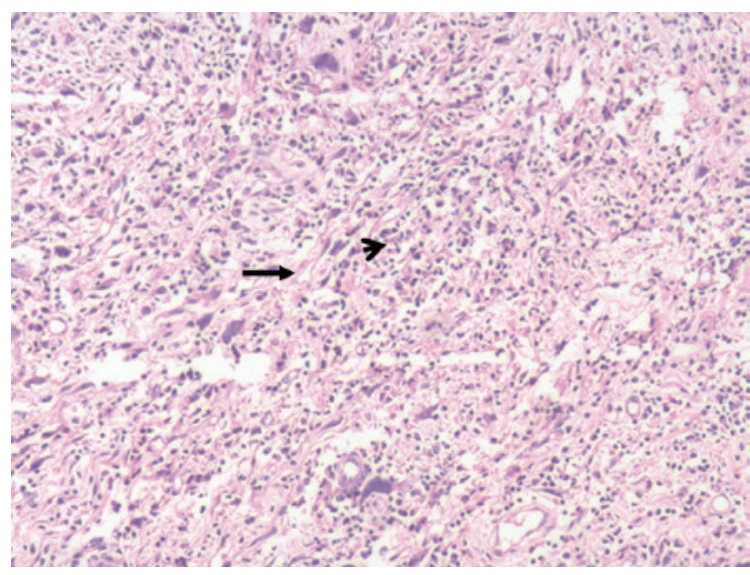

Figure 3. Microscopically the tumor exhibited proliferation of spindle cells (arrow) and infiltration of lymphocytes, plasma cells and eosinophils (arrowhead). Hematoxylin and eosin staining; magnification, x100.

The clinical presentation of IMT is non-specific and may include fever, weight loss, malaise, anemia, thrombocytosis, polyclonal hyper-globulinemia and/or elevated erythrocyte sedimentation rate (2). Frequently, IMT induces anatomical site-specific presentations. Retroperitoneal IMT typically grows slowly and may present as a solid abdominal mass, accompanied by abdominal pain and weight loss. Gastrointestinal and urinary symptoms, particularly bowel and ureteral obstruction, respectively, may occur with an enlarging expansile mass (2). The patient in the present report was asymptomatic with the primary tumor, while the recurrence presented with a palpable mass but an unremarkable hematological and biochemical profile.

There are no radiographic features specific to IMT. In abdominal IMT, a solid mass abutting and compressing various organs is the typical presentation, frequently accompanied by obstruction or invasion of the affected organ(s). CT and magnetic resonance imaging may identify a homogeneous or heterogeneous, hypodense or isodense lesion. Enhancement may be variable and may reveal central necrosis or fibrosis (13).

The diagnosis of IMT mainly occurs via pathological examination. Macroscopically, IMT may be firm, fleshy or gelatinous, with a white or tan cut surface. The tumors vary in size, reaching up to $20 \mathrm{~cm}$ in the greatest dimension. Histologically, IMT is characterized by spindle cell proliferation with a prominent inflammatory infiltrate. IMT lesions are also positive for vimentin, smooth muscle actin, muscle-specific actin and occasionally, desmin and cytokeratin (12). 
IMT is a neoplasm with 'intermediate biological potential' with a propensity for local recurrence, while rarely metastasizing. In a series of 84 cases of extrapulmonary IMT, recurrence was reported in $25 \%$ and distal metastasis in only $5 \%$ of cases (12). Histopathological features alone may not be sufficient for the prediction of malignant transformation, as tumor size, cellularity, mitotic activity and the presence of necrosis are not significantly correlated with risk of metastasis. However, nuclear atypia and ganglion-like cells may indicate more aggressive cellular behavior. ALK-positive tumors have a notably low risk of metastasis, and ALK reactivity has not been found to correlate with recurrence (12). Compared with other extra-pulmonary lesions, retroperitoneal IMTs tend to be more aggressive. Of the 12 reported cases in the English literature, summarized in Table I, 3 cases developed metastases and one exhibited local recurrence, with follow-up periods varying from 3 to 80 months (2-10).

The mainstay of management of IMT, as previously reported, is by surgical excision for definitive or palliative treatment (2-10). Incomplete tumor removal frequently results in local recurrence (3), which was likely the case in the present patient. The patient underwent a second successful re-resection, with no recurrence at 30 months follow-up. However, this follow-up period is relatively short and vigilant monitoring is required to facilitate rapid detection of further recurrence.

Tao et al (9) reported an unresectable retroperitoneal IMT, which was successfully treated with methotrexate, cisplatin and diclofenac sodium, facilitating maintenance of a clinical 'free of disease' status for 3 years. Przkora et al (4) also reported a case of unreseactable IMT, treated with anti-inflammatory agent, ibuprofen, where the patient was 'stabilized' for 12 months. The possible role of the ALK gene in IMT pathogenesis highlights the potential for use of a tyrosine-kinase inhibitor in a neoadjuvant or adjuvant capacity (1). Furthermore, a study reported a sustained partial response to the ALK-inhibitor, crizotinib, in a patient with an ALK-translocated IMT (14). The role of radiotherapy in IMT is unknown, although it may have potential benefits, particularly in unresectable cases. To date, there is no established protocol for the treatment of IMT, due to its rarity $(9,15)$.

Retroperitoneal IMT is a rare entity with unclear etiology and non-specific clinical symptoms. Precise diagnosis mainly occurs via histopathological analysis. Complete surgical excision, where feasible, is the recommended treatment. In addition, complete surgical re-resection is also recommended for the treatment of recurrent lesions.

\section{References}

1. Cessna MH, Zhou H, Sanger WG, Perkins SL, Tripp S, Pickering D, Daines C and Coffin CM: Expression of ALK1 and p80 in inflammatory myofibroblastic tumor and its mesenchymal mimics: A study of 135 cases. Mod Pathol 15: 931-938, 2002

2. Chatzikokolis S, Troupis TG, Michalinos A, Bafaloukas N, Filippidis T and Gennimatas V: Retroperitoneal inflammatory myofibroblastic tumor. Am Surg 78: E190-E191, 2012.

3. Coffin CM, Watterson J, Priest JR and DehnerLP: Extrapulmonary inflammatory myofibroblastic tumor (inflammatory pseudotumor). A clinicopathologic and immunohistochemical study of 84 cases. Am J Surg Pathol 19: 859-872, 1995.

4. Przkora R, Bolder U, Schwarz S, Jauch KW, Spes J, Andreesen R and Mackensen A: Regression of nonresectable inflammatory myofibroblastic tumours after treatment with nonsteroidal anti-inflammatory drugs. Eur J Clin Invest 34: 320-321, 2004.

5. Attili SV, Chandra CR, Hemant DK, Bapsy PP, RamaRao C and Anupama G: Retroperitoneal inflammatory myofibroblastic tumor. World J Surg Oncol 3: 66, 2005.

6. Coffin CM, Hornick JL and Fletcher CD: Inflammatory myofibroblastic tumor: Comparison of clinicopathologic, histologic and immunohistochemical features including ALK expression in atypical and aggressive cases. Am J Surg Pathol 31: 509-520, 2007.

7. Koirala R, Shakya VC, Agrawal CS, Khaniya S, Pandey SR, Adhikary S and Pathania OP: Retroperitoneal inflammatory myofibroblastic tumor. Am J Surg 199: e17-e19, 2010.

8. Ziadi S, Trimeche M, Mestiri S, Boujelbene N, Mokni M, Sriha B and Korbi S: Retroperitoneal myofibroblastic inflammatory tumor. Tunis Med 89: 400-401, 2011.

9. Tao YL, Wang ZJ, Han JG and Wei P: Inflammatory myofibroblastic tumor successfully treated with chemotherapy and nonsteroidals: A case report. World J Gastroenterol 18: 7100-7103, 2012.

10. Mali VP, Tan HC, Loh D and Prabhakaran K: Inflammatory tumour of the retroperitoneum - a case report. Ann Acad Med Singapore 34: 632-635, 2005.

11. Gómez-Román JJ, Sánchez-Velasco $P$, Ocejo-Vinyals G, Hernández-Nieto E, Leyva-Cobián F and Val-Bernal JF: Human herpesvirus- 8 genes are expressed in pulmonary inflammatory myofibroblastic tumor (inflammatory pseudotumor). Am J Surg Pathol 25: 624-629, 2001.

12. Gleason BC and Hornick JL: Inflammatory myofibroblastic tumours: Where are we now? J Clin Pathol 61: 428-437, 2008.

13. Aptel S, Gervaise A, Fairise A, Henrot P, Leroux A, Guillemin F, Laurent V and Régent D: Abdominal inflammatory myofibroblastic tumour. Diagn Interv Imaging 93: 410-412, 2012.

14. Butrynski JE, D'Adamo DR, Hornick JL, Dal Cin P, Antonescu CR, Jhanwar SC, Ladanyi M, Capelletti M, Rodig SJ, Ramaiya N, et al: Crizotinib in ALK-rearranged inflammatory myofibroblastic tumor. N Engl J Med 363: 1727-1733, 2010.

15. Chavez C and Hoffman MA: Complete remission of ALK-negative plasma cell granuloma (inflammatory myofibroblastic tumor) of the lung induced by celecoxib: A case report and review of the literature. Oncol Lett 5: 1672-1676, 2013. 